\title{
Bilateral Pulmonary Embolism Associate with Varicella Zoster Meningoencephalitis in an Immunocompetent Adult: A Case Report and Literature Review
}

\author{
Alexandra Gillispie, BS ${ }^{1}$, Sofanit Dessie, MD ${ }^{1}$, Rodrigo Aguilar, MD ${ }^{1}$, \\ Kemnasom Nwanwene, MD' ${ }^{1}$, Ghassan Bandak, MD' ${ }^{1}$ Elie Khalil, MD', \\ Imran Khawaja, MD'
}

ABSTRACT

The association of varicella-zoster virus (VZV) with a considerable variety of complications has been well documented throughout literature. Primary infection, most often seen in children, is generally characterized by mild symptoms, as opposed to the more severe presentations in adult and immunocompromised populations. Manifestations of disseminated VZV include dermatologic, pulmonary, neurologic, and ocular involvement. Vascular and hematologic complications are also reported and sometimes pose serious health threats. We present an interesting case of disseminated VZV infection, complicated by meningitis, encephalitis, and bilateral pulmonary embolism in an otherwise immunocompetent 52-year-old man with no prior chicken pox exposure.
Author affiliations are listed at the end of this article.

Correspondence to: Alexandra Gillispie, BS Marshall Unviersity Joan C. Edwards School of Medicine gillispie44@marshall.edu

\section{KEYWORDS}

Varicella, Pulmonary Embolism, Lupus Anticoagulant

\section{INTRODUCTION}

Primary varicella infection, caused by VZV, has historically been considered a disease of childhood or immunocompromised adults. It is spread through droplet or airborne transmission, and the usual incubation period is from 11-20 days. ${ }^{1}$ Central nervous system complications, such as vasculopathy and thrombotic events, can follow both primary infection and reactivation of VZV. ${ }^{2}$ Prior case reports have reported post-VZV thrombotic complications in children with proposed mechanisms including vasculitis, direct endothelial damage, and acquired protein $S$ deficiency secondary to the development of antibodies to the protein. ${ }^{3}$

Reports of thrombotic complications due to VZV are not common in medical literature. They include cases of cortical sinus venous thrombosis, lower extremity deep vein thrombosis, and a few cases of pulmonary embolism. ${ }^{4,5}$ We present an interesting case of disseminated VZV infection complicated by neurologic and hematologic complications in an immunocompetent patient, hoping to shed light and bring attention to this potentially life-threatening association.

\section{CASE DESCRIPTION}

A 52-year-old Caucasian male with a past medical history of hyperlipidemia and obesity (body mass index of 36) was transferred to the intensive care unit for evaluation of a rash and altered mental status. His symptoms began five days prior to admission. The patient was complaining of flulike symptoms, including fever, nausea, vomiting, headache, and large localized painful lesions in the posterior occipital area. He had no prior exposure to chickenpox. He was a former smoker, consumed alcohol socially, and worked as a welder. His only history of travel was to Mexico twenty-five years ago. Notably, the patient's father-in-law, who was immunocompromised secondary to cancer, had a 
similar rash three weeks prior before passing away. On presentation, blood pressure was $142 / 95 \mathrm{mmHg}$, heart rate was 53 beats per minute, respiratory rate was 18 breaths per minute, temperature was $38.50 \mathrm{C}$, and oxygen saturation was $95 \%$ on room air. On clinical examination, he was difficult to arouse, scored 9/15 on the Glasgow Coma Scale, and had non-purposeful movement of all extremities. He also had diffuse papulovesicular lesions on the face, trunk, arms, legs, and pubic areas in different stages of evolution. Meningeal signs were difficult to assess due to changes in mentation.

His chest, cardiac, and abdominal exams were otherwise unremarkable. Initial laboratory investigations revealed white blood cell count (WBC) of $10.6 \times 109$ cells/liter (82\% neutrophils), creatine of $1.04 \mathrm{mg} / \mathrm{dL}$, lactic acid of $1.6 \mathrm{mmol} / \mathrm{L}$, aspartate aminotransferase of $56 \mathrm{U} / \mathrm{L}$, and alanine aminotransferase of $106 \mathrm{U} / \mathrm{L}$. The urine analysis, urine drug screen, human immunodeficiency virus antibody, Lyme titers, rapid plasma reagin, acute hepatitis panel, and influenza panel were all negative. An abdominal ultrasound indicated fatty infiltration of the liver but no features of cirrhosis. Due to concern for disseminated VZV and meningoencephalitis, the patient was immediately placed in airborne precautions and started on therapy with ceftriaxone, vancomycin, ampicillin, and intravenous (IV) acyclovir. A swab from his draining vesicles was sent for testing and came back positive for VZV.

Initial head computed tomography (CT) was negative for any acute intracranial process, and chest $\mathrm{x}$-ray gave no indication of acute pulmonary or cardiac processes. Due to deteriorating mental status, the patient was intubated and started on mechanical ventilation for airway protection. A lumbar puncture was obtained and yielded yellow cerebrospinal fluid with a glucose of 32 , protein of 528 , and WBC of 551 (neutrophils-15, lymphocytes62). Polymerase chain reaction analysis of the fluid was positive for VZV.

A magnetic resonance imaging (MRI) of the brain was performed with and without contrast and showed extensive abnormal leptomeningeal FLAIR signal consistent with meningoencephalitis and associated cerebritis in the right temporal lobe. Magnetic resonance angiogram did not reveal cerebral vasculitis. (Figures $1 \mathrm{a}$ and $1 \mathrm{~b}$ ).
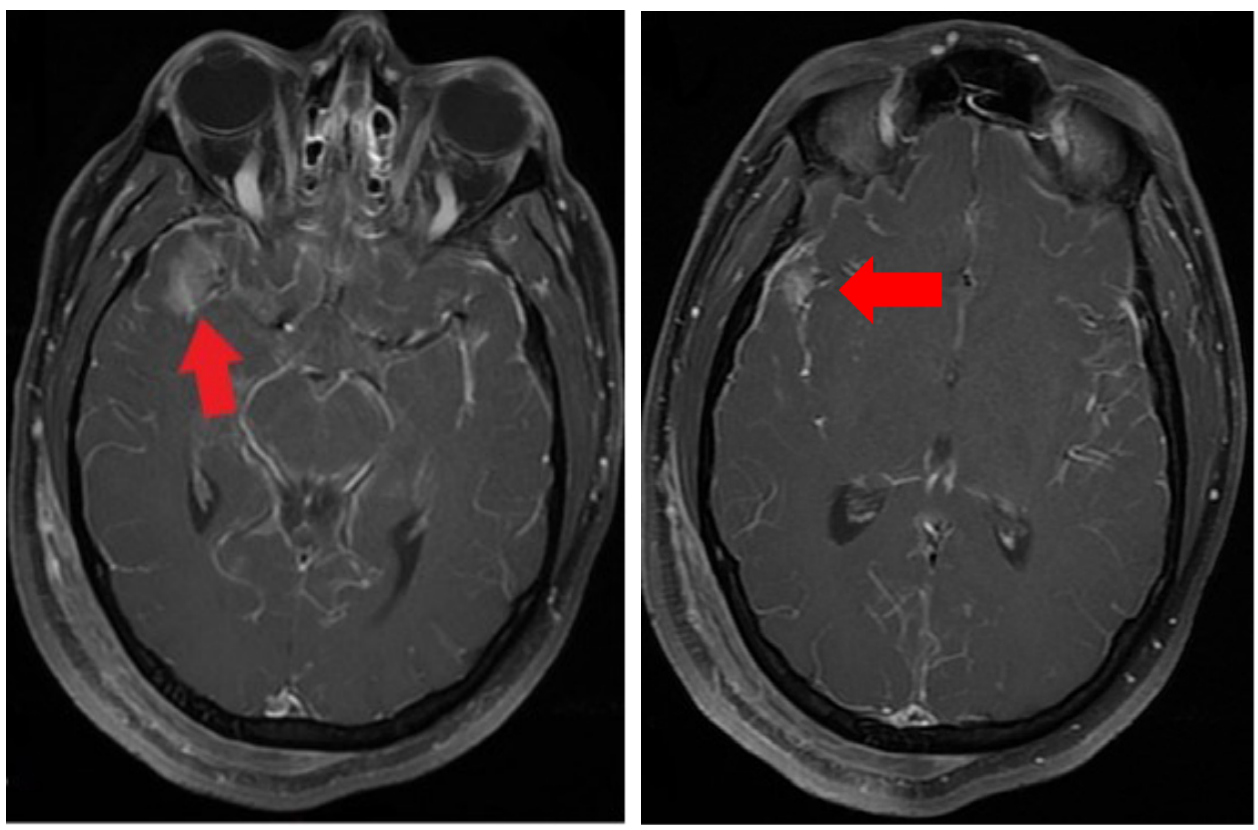

FIGURES 1A \& 1B: MRI of the brain showing extensive abnormal sulcal FLAIR and diffuse enhancement with ill-defined increased signal in the anterior aspect of the right temporal lobe (as indicated by the arrows). 
On day 2, a punch skin biopsy was obtained from the active skin lesions, and it revealed necrotic keratinocytes suggestive of herpes viral cytopathic changes including multi-nucleation and chromatin margination (Figures $2 \mathrm{a}, 2 \mathrm{~b}$, and $2 \mathrm{c}$ ).

After 24 hours of mechanical ventilation, the patient's mental status improved, but an attempt to wean him failed due to worsening hypoxia and increased oxygen requirements. The electrocardiogram showed sinus tachycardia with no features of acute ischemia. Transthoracic echocardiogram was obtained which revealed normal ventricular function without any major valve disease, wall motion abnormality, or intracardiac shunting. CT angiogram (CTA) of the chest revealed bilateral acute pulmonary emboli and lower lobe infiltrates versus atelectasis (Figure 3 ).

The patient was started on anticoagulation therapy with therapeutic unfractionated heparin. Lower extremity doppler was negative for deep vein thrombosis (DVT). Hypercoagulable work-up was subsequently performed, including ATIII, Factor V
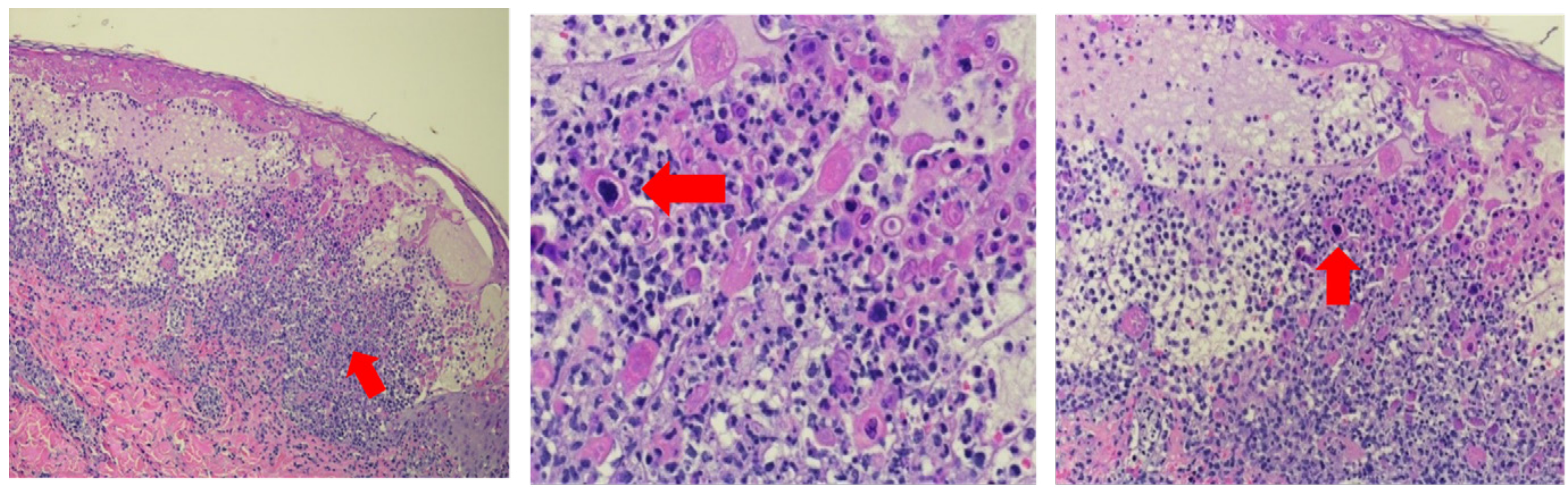

FIGURES 2A, 2B, 2C: Skin biopsy showing acantholytic keratinocytes (indicated by the arrows) with characteristic margination of the nuclear chromatin, multinucleation, and nuclear inclusion bodies.

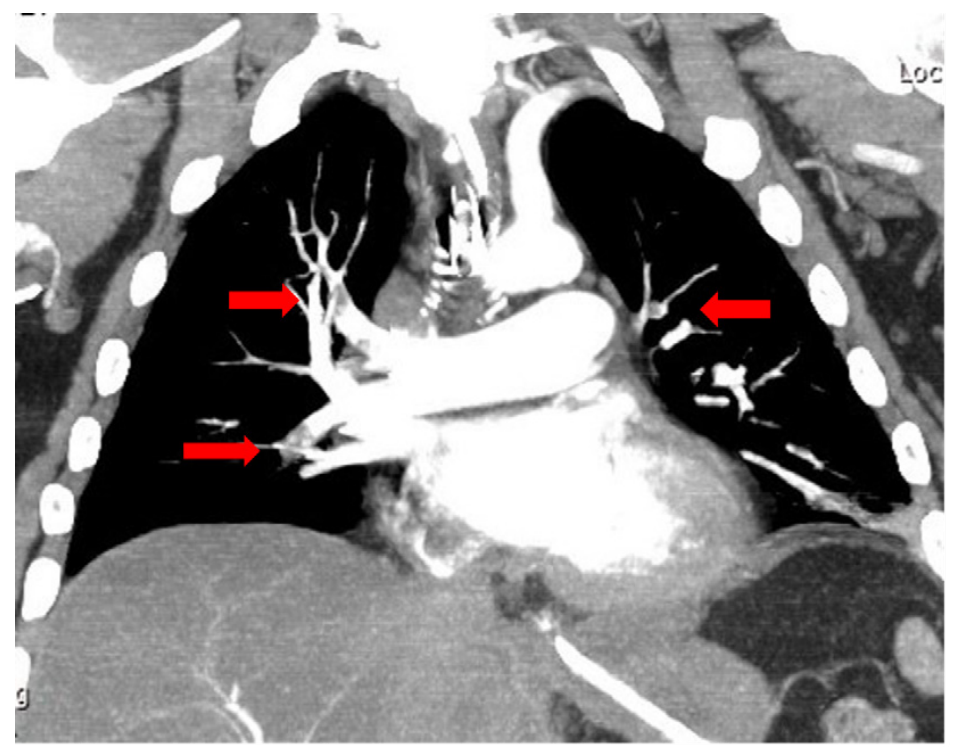

FIGURE 3: CT of the chest with IV contrast showing pulmonary emboli (as indicated by the arrows) involving bilateral pulmonary arteries. 
Leiden, Proteins $C$ and S, IgM/ IgG anticardiolipin, IgM/lgG beta-2-glycoprotein, and anti-neutrophil cytoplasmic antibody levels. All were reported to be in the normal range other than a mildly elevated lupus anticoagulant level.

The patient was removed from mechanical ventilation on day three. On day seven of hospitalization, he was discharged home on apixaban and oral valacyclovir to complete twentyone days course of treatment. He was advised to follow up with his primary care physician to complete an evaluation for hypercoagulability, including age-appropriate cancer screening, as well as follow up with hematology with the plan of repeating the hypercoagulable work up in three months to determine the necessary duration of anticoagulation.

\section{DISCUSSION}

Prior to the advent of the varicella vaccine in 1995, national data reported that more than $95 \%$ of Americans were seropositive before age 20.4 Even though the vaccine has drastically reduced the number of patient cases, chickenpox remains an extremely contagious virus, reportedly leading to the secondary transfer of infection in exposed susceptible individuals at a rate of greater than $90 \%{ }^{6}$ VZV infection initially causes viremia, allowing the virus to eventually manifest in a variety of systemic complications. ${ }^{7}$ Micro-thrombotic events are not considered to be uncommon, but, as with all varicella associations, are more often seen in children. Rabah et al provided a literature review of DVTs occurring in children with a recent history of varicella infection. ${ }^{5}$ We could only find five other cases involving otherwise unprovoked pulmonary embolism in immunocompetent adults with no underlying comorbidities or risk factors for a hypercoagulable state, as seen in Table 1. Of those, two were evaluated for the presence of antibodies with only one producing significant findings. The other studies credited the thrombotic state to endothelial damage related to the viral infection..$^{8-12}$

The etiology of post-varicella thrombosis is considered multifactorial, attributed to several pathophysiologic mechanisms including immunemediated deficiency of natural anticoagulants, the transient formation of lupus anticoagulant/ antiphospholipid syndrome, and direct endothelial damage. ${ }^{13}$ Virologic analysis has shown VZV is capable of direct arterial infection and subsequent pathologic transmural vascular remodeling. ${ }^{2}$

Transient protein $\mathrm{S}$ deficiency has commonly been cited as a causal source for a prothrombotic state. It is postulated that this occurs through the induction of anti-protein S autoantibodies secondary to molecular mimicry. ${ }^{14}$ Other antibodies, which create a pseudo-antiphospholipid syndrome, are seen with increased frequency in multiple viral infections, but, whether due to downregulation of immune mediators or antibody concentration, they seem to lead to more significant vasculopathy events in VZV

\begin{tabular}{|c|c|c|c|c|}
\hline Author & Year & Age & Gender & Antibody presence \\
\hline Gogos CA et al & 1993 & 36 & M & Not performed \\
\hline Viseux et al & 2000 & 29 & M & aCL, IgM anti- $\beta 2-G P I$ \\
\hline Dahan et al & 2005 & 34 & M & Not performed \\
\hline Paul G et al & 2016 & 37 & M & Not performed \\
\hline Khan et al & 2019 & 26 & M & Not performed \\
\hline
\end{tabular}

TABLE 1: Reported cases of pulmonary embolism following VZV infection in adults with no other apparent cause for hypercoagulability Abbreviations: aCL: anticardiolipin; IgM anti- $\beta 2-G P I$ : Immunoglobulin $M$ anti $\beta 2$ glycoprotein 
infections. $^{15}$

Initial coagulation workup in our patient was significant for an increase in lupus anticoagulant (LA). Deficiencies in protein $\mathrm{S}$ were not seen though this could be due to the initiation of a heparin drip prior to taking the labs. Although there was concern that heparin could also cause falsely elevated antibody values, Genzen and Miller previously asserted that use of heparin did not affect LA results evaluated through the DRVVT testing system because it contains an anti-heparin agent.16 Furthermore, preliminary labs did not indicate concern for a hypercoagulable state with activated partial thromboplastin time and platelet values both being within normal limits. The prognosis of thrombosis associated with VZV is noted to be good but hypercoagulable workup after recovery is advisable in order to diagnose any hereditary prothrombotic states and determine the necessary duration of anticoagulation.

\section{CONCLUSION}

Thrombotic complications secondary to VZV can include life-threatening conditions such as pulmonary embolism. Physicians should keep a high index of suspicion for such complications as they are potentially lethal.

\section{AUTHOR AFFILIATIONS}

1. Marshall University Joan C. Edwards School of Medicine, Huntington, West Virginia

\section{REFERENCES}

1. Choo PW, Donahue JG, Manson JE, Platt R. The epidemiology of varicella and its complications. J Infect Dis. 1995;172(3):706-12.

2. Nagel M, Gilden D. Editorial commentary: varicella zoster virus infection: generally benign in kids, bad in grown-ups. Clin Infect Dis. 2014;58(11):1504-6.

3. Heininger U, Seward JF. Varicella. Lancet. 2006;368(9544):1365-76.

4. Kelley PW, Petruccelli BP, Stehr-Green P, Erickson
RL, Mason CJ. The susceptibility of young adult Americans to vaccine-preventable infections. A national serosurvey of US Army recruits. JAMA. 1991;266(19):2724-9.

5. Rabah F, El-Banna N, Abdel-Baki M, Beshlavi I, Macaraig D, Bhuyan D, et al. Postvaricella thrombosis-report of two cases and literature review. Pediatr Infect Dis J. 2012;31(9):985-7.

6. Gabutti G, Bolognesi N, Sandri F, Florescu C, Stefanati A. Varicella zoster virus vaccines: an update. Immunotargets Ther. 2019;8:15-28.

7. Moffat J, Ku CC, Zerboni L, Sommer M, Arvin A. VZV: pathogenesis and the disease consequences of primary infection. In: Arvin A, Campadelli-Fiume G, Mocarski E, Moore PS, Roizman B, Whitley R, et al., editors. Human Herpesviruses: Biology, Therapy, and Immunoprophylaxis. Cambridge2007.

8. Gogos CA, Apostolidou E, Bassaris HP, Vagenakis AG. Three cases of varicella thrombophlebitis as a complication of varicella zoster virus infection. Eur J Clin Microbiol Infect Dis. 1993;12(1):43-5.

9. Viseux V, Darnige L, Carmi E, Chaby G, Poulain JF, Cevallos R, et al. Pulmonary embolism and transitory anti-beta2-GPI antibodies in an adult with chicken pox. Lupus. 2000;9(7):558-60.

10. Dahan E, Simsolo C, Merei M, Vigder F, Tatoor I, Blum A. Varicella zoster infection and pulmonary complications. Eur J Intern Med. 2005;16(6):44950.

11. Khan R, Yasmeen A, Pandey AK, Al Saffar K, Narayanan SR. Cerebral Venous Thrombosis and Acute Pulmonary Embolism following Varicella Infection. Eur J Case Rep Intern Med. 2019;6(10):001171.

12. Paul G, Paul BS, Singh G. Unseen face of varicellazoster infection in adults. Indian J Crit Care Med. 2016;20(12):731-4.

13. Josephson C, Nuss R, Jacobson L, Hacker MR, Murphy J, Weinberg A, et al. The varicellaautoantibody syndrome. Pediatr Res. 2001;50(3):345-52.

14. Sardana V, Mittal LC, Meena SR, Sharma D, $\mathrm{Khandelwal} \mathrm{G}$. Acute venous sinus thrombosis after chickenpox infection. J Assoc Physicians India. 2014;62(8):741-3.

15. Avcin T, Toplak N. Antiphospholipid antibodies in response to infection. Curr Rheumatol Rep. 2007;9(3):212-8.

16. Genzen JR, Miller JL. Presence of direct 
thrombin inhibitors can affect the results and interpretation of lupus anticoagulant testing. Am J Clin Pathol. 2005;124(4):586-93. 